\title{
Do "Economic" Institutions Influence on Monetary Stability and Economic Growth?
}

\author{
Andrey Kolomiets \\ The Institute of Economy \\ Russian Academy of Sciences (RAS) \\ Moscow, Russia \\ agk1807@mail.ru
}

\begin{abstract}
The Author investigates the problem of mutual influence and dependences between estimates of quality of "economic" institutions, indicators of monetary stability, and growth rates in representative group of different economies developed, developing and transitional (Selected Economies) at 2002-2013, using methods of ranking and statistical correlation. In developed economies these dependences most strong. In selected developing and transitional economies the dependences between growth rates, quality of "economic" institutions, and monetary stability were statistically weak or not significant at studied period. These results put before need to investigate a complex of questions about mutual influence of quality of institutional environment, monetary stability, and economic growth in the developing and transitional economies more detail. Obviously it's expedient to consider not the growth rates only, but first of all, quality of growth and to answer whether is the best for the developing and transitional economies the same parameters of dynamics of inflation and money supply which are recognized optimal at advanced economies?
\end{abstract}

Keywords-Institutions quality; Monetary stability; Inflation; Growth rates; Developing and transitional economies

\section{INTRODUCTION}

In common opinion the rise of the institutional environment quality and the restricting of inflation are the main bases of steady growth and purposes of economic governance in the developing and transitional economies. Influence of institutions on growth is one of the main topics of modern economic discussions [1], [2].

Meanwhile the experience of different developing and transitional economies can give arguments for various views about mutual influence of institutions quality, monetary stability, and growth. Relatively high inflation, or fast expansion of money's quantity, or high banking lending rates, or all together - main features of monetary instability or (the same, but more exactly) of the monetary sphere's instability were noted during one and a half decade after 2000 at the majority of the developing and transitional economies, especially at economies with low quality of institutional environment. At the same time part of the developing and transitional economies are distinguished by a higher estimation of institutions, more stable condition of the monetary sphere, and steady growth of GDP.
We investigates dependences between estimates of quality of "economic" institutions (definition see below), indicators of monetary stability, and growth rates in representative group of different economies - developed, developing and transitional (Selected Economies) at 2002-2013, using methods of ranking and statistical correlation.

The paper is structured as follows. Section II includes preliminary notes about: the Selected Economies, studied period, key indicators used as tools of analyses, and the main steps of the investigation. Section III examines statistically significant dependences between estimations: quality of "economic" institutions, monetary sphere's stability, and GDP growth rates at Selected Economies (are presented at Appendix). Section IV summarizes some conclusions and questions for further studies.

\section{PRELIMINARY NOTES}

The time period under investigation is 2002-2013. At the beginning of this period at considerable number of economies a long growth took place. After 2008 rates of growth at the majority of developing and transitional economies had decreased. Hence, average annual indicators at 2002-2013 reflect multidirectional changes of an economic environment. Thus the using of these average annual indicators excluded the salient influence of short-term markets volatility on dynamics of prices and GDP.

We consider necessary and expedient to compare institutions quality, monetary sphere's stability/instability, and growth rates of the largest developed, developing and transitional economies in which endogenous factors of the institutional environment, prices change and GDP growth play a leading role. Therefore Selected Economies are separated from other economies by criteria of considerable volume of GDP, considerable population, capacious domestic market, sovereignty and relative independence of the monetary system. The result of the selection includes 25 developed, developing and transitional largest economies of Europe, North and South America, Asia and Australia. Owing to incompleteness of initial data the selection didn't include many large economies: Pakistan, Poland, Germany and others.

The fullest and adequate system of an estimation of institutions quality is Worldwide Governance Indicator (WGI). WGI mainly characterizes national administrative and 
government agencies which determine the environment for economic activities and the ability of these agencies to deliver a level playing field for all economic actors [3]. In order to find the most accurate base for estimations for purposes of this study we were to be addressed to WGIestimations characterizing quality of regulation of economic agents' activity. We rely on classification of Institutions "as being legal, political, economic, and social institutions" [4]. "Economic" institutions ("Government Effectiveness", "Regulatory Quality", and "Rule of Law") directly are securing a properly working market, such as the legal system enforcement of property rights, some parts of regulation, etc., were separated by us from other sets of WGIestimations which characterize primarily political and social aspects of institutions functioning. Average annual percentiles' ranks of "Government Effectiveness" (GE), "Regulatory Quality"(RQ), and "Rule of Law"(RL) were appropriated to each Selected Economy depending on its place in order of assessments. The sum of above ranks is defined as Rank of "Quality of Economic Institutions" (QEI Rank) of each economy (Table I).

We marked key indicators of the monetary sphere's stability. For this purpose we use Consumer Price Index (CPI) as "inflation" index; Index of Broad Money (BM - Money and quasi money) as index "changes of quantity of money"; and Index of Lending Interest Rates (LIR) as "interest rates" index. (Data about Broad Money in Netherlands and Italy - is the same as Euro Area; Netherlands' LIR - no data at 20122013; Turkey LIR - data on rates for loans granted by Central Bank of Turkey are used). Ranks were appropriated to each economy also depending on a place in order of assessments. The sum of above ranks is defined as Rank of "Monetary Sphere's Stability" (MSS Rank) (Table I).

At next step of the investigation we compared ranks (and places in general orders) of each of Selected Economies by criteria of Monetary Sphere's Stability (MSS), by criteria of Quality of Economic Institutions (QEI), and by criteria of GDP average annual rates at studied period. Finally we tried to value the dependence (the correlation) between above groups of ranks. Results of the ranging and correlation coefficients are presented at Table II. This method is extremely simple and may be tested; it is difficult to interpret results of these calculations in other way which differs from the interpretation presented.

\section{FinAL RESULtS}

In total of Selected Economies strong dependence between quality of "economic" institutions and monetary phenomena at the period 2002-2013 is confirmed by correlation coefficient $(\mathrm{R} 1=0,820167)$ between two sets of variables: Ranks of "Quality of "Economic" Institutions" and Ranks of "Monetary Sphere's Stability". At improvement of Quality of Economic Institutions (QEI Ranks are lower) monetary sphere is more stable (MSC Rank is lower) and vice versa, if QEI Ranks are higher, monetary sphere is more unstable as a rule. For the developing and transitional Selected Economies (all except developed economies - USA, Britain, Netherlands, Italy, and Japan which distinguish relative low growth rates, relative stability of the monetary sphere, and high - except Italy - quality of "economic" institutions), the correlation coefficient $(\mathrm{R} 2=0,717398)$ between MSS Ranks and QEI Ranks confirms that in the developing and transitional economies differing by less quality of institutes in general, noted dependence is also rather considerable.

In total of Selected Economies the dependence between Ranks of stability of monetary sphere (MSS) and average annual GDP rates (at Table II ranged from a maximum to a minimum) existed at studied period, but not as strong; it confirms by correlation coefficient $\mathrm{R} 3=0,547201$. The exception of above five developed economies has the result minimization of statistically significant dependence between Ranks of stability of the monetary sphere (MSS) and average annual GDP rates at studied period R4 $=0,107512$. Thus, for group of the developing and transitive economies presented in Selected Economies this dependence didn't be significant.

The correlation coefficient between QEI Ranks and average annual GDP rates for all Selected Economies at studied period - R5 $=0,558698$. The exception of above five economies has the result - weak statistically significant dependence between average annual GDP rates and quality of "economic" institutions - R6 = 0,313993.

\section{SOME CONCLUSIONS.}

Presented results are confirming that mutual dependences between estimations of "economic" institutions quality, monetary sphere's stability, and growth rates exist in different developed, developing and transitional economies. In developed economies these dependences most strong. In selected developing and transitional economies the dependences between growth rates and "economic" institutions quality and monetary sphere's stability were statistically weak or not significant. Thus other dependences had priority influence on growth rates in these economies at studied period.

Apparently, mutual influence between quality of institutional environment, monetary stability, and growth in the developing and transitional economies is more complex, than it often is considered. Results of this study puts before need to investigate these questions in more detail. In particular as the result of influence of stability and quality of institutional environment it's expedient to consider not the growth rates only, but first of all quality of growth. The experience of advanced economies supports such point of view. Besides it is necessary to remember about distinctions between real "economic" institutions quality, including many informal rules, and WGI estimations, which primarily centered on law norms and formal written rules. Such distinctions are famous in the developing and transitional economies and the influence of informal rules for contract stability and rise of production may be positive and also negative in these economies. Also, so far there is without consideration the question: whether is the best for the developing and transitional economies in conditions of fast changes of their structure and institutional transformations the same parameters of dynamics of inflation and money supply which are recognized optimal at advanced economies. 


\section{REFERENCES}

[1] E.L. Glaeser, R. LaPorta, F. Lopez-de-Silanes, and A. Shleifer, Do Institutions Cause Growth? Cambridge, MA, NBER 2004 (Working Paper 10568).

[2] K Masuch, E. Moshammer, and B. Pierluidi, Institutions, Public Debt \& Growth in Europe. ECB. Working Paper Series, No.1963, 14 Sept. 2016.
[3] D. Kaufmann, A. Kraay, and M. Mastruzzi, The Worldwide Governance Indicators: Methodology and Analytical Issues, World Bank Research Working Paper, No.5430, 2010.

[4] P.L. Joskow, "Introduction to New Institutional Economics: A Report Card," in E. Brousseau and J.-M. Glachant (eds.), New Institutional Economics: a Guidebook, Cambridge/New York: Cambridge University Press, 2008, pp. 1-19.

[5] Data sources: World Bank statistics and Bank Worldwide Governance Indicators (www.worldbank.org).

\section{APPENDIX}

TABLE I. SELECTED ECONOMIES RANKS

\begin{tabular}{|c|c|c|c|c|c|c|c|c|c|}
\hline \multicolumn{10}{|c|}{$\begin{array}{l}\text { Selected Economies "Monetary Sphere's Stability (MSS)" } \\
\text { and "Quality of "Economic" Institutions (EIQ)" Ranks [5]. }\end{array}$} \\
\hline \multirow[t]{3}{*}{$\begin{array}{l}\text { Num. of } \\
\text { Place }\end{array}$} & \multirow[t]{3}{*}{ Economy } & \multicolumn{4}{|c|}{$\begin{array}{c}\text { MSS Average Annual Rank } \\
(2002-2013)\end{array}$} & \multicolumn{4}{|c|}{$\begin{array}{c}\text { EIQ Average Annual Rank } \\
(2002-2013)\end{array}$} \\
\hline & & CPI & $B M$ & $L I R$ & $\begin{array}{c}\text { Overall / } \\
\text { Rank }\end{array}$ & $G E$ & $R Q$ & $R L$ & $\begin{array}{c}\text { Overall// } \\
\text { Rank }\end{array}$ \\
\hline & & $\begin{array}{l}\text { Data/ } \\
\text { Place }\end{array}$ & $\begin{array}{l}\text { Data/ } \\
\text { Place }\end{array}$ & $\begin{array}{l}\text { Data/ } \\
\text { Place }\end{array}$ & $(3+4+5)$ & $\begin{array}{l}\text { Data/ } \\
\text { Place }\end{array}$ & $\begin{array}{l}\text { Data/ } \\
\text { Place }\end{array}$ & $\begin{array}{l}\text { Data/ } \\
\text { Place }\end{array}$ & $(7+8+9)$ \\
\hline 1 & 2 & 3 & 4 & 5 & 6 & 7 & 8 & 9 & 10 \\
\hline 1 & Japan & $\begin{array}{l}-0,2 \\
/ 1\end{array}$ & $\begin{array}{ll}1,3 \\
/ 1\end{array}$ & $\begin{array}{l}1,7 \\
/ 1 \\
\end{array}$ & 3 & $\begin{array}{l}88,9 \\
15\end{array}$ & $\begin{array}{l}82,2 \\
15\end{array}$ & $\begin{array}{l}87,4 \\
15\end{array}$ & 15 \\
\hline 2 & $\begin{array}{ll}\text { Euro Area } & \\
\text { Netherlands }\end{array}$ & $\begin{array}{l}1,9 \\
12\end{array}$ & $\begin{array}{l}9,4^{* *} \\
16\end{array}$ & $\begin{array}{l}2,6^{*} \\
12\end{array}$ & 10 & $\begin{array}{l}96,2 \\
11\end{array}$ & $\begin{array}{l}97,4 \\
/ 1\end{array}$ & $\begin{array}{l}96,1 \\
/ 1\end{array}$ & 3 \\
\hline 3 & United States & $\begin{array}{ll}2,3 \\
14\end{array}$ & $\begin{array}{ll}5,8 \\
12\end{array}$ & $\begin{array}{l}4,8 \\
14\end{array}$ & 10 & $\begin{array}{l}91,4 \\
14\end{array}$ & $\begin{array}{l}92,1 \\
14\end{array}$ & $\begin{array}{l}91,7 \\
14\end{array}$ & 12 \\
\hline 4 & Great Britain & $\begin{array}{l}2,5 \\
16 \\
\end{array}$ & \begin{tabular}{|l}
7,4 \\
13 \\
\end{tabular} & $\begin{array}{l}2,8 \\
13 \\
\end{array}$ & 12 & $\begin{array}{l}92,6 \\
13 \\
\end{array}$ & $\begin{array}{l}96,6 \\
12 \\
\end{array}$ & $\begin{array}{l}93,4 \\
13 \\
\end{array}$ & 8 \\
\hline 5 & Euro Area - Italy & $\begin{array}{l}2,2 \\
13 \\
\end{array}$ & $\begin{array}{l}9,4^{* *} \\
17 \\
\end{array}$ & \begin{tabular}{|l|l}
5,5 \\
15 \\
\end{tabular} & 15 & $\begin{array}{l}68,7 \\
19 \\
\end{array}$ & $\begin{array}{l}77,7 \\
16 \\
\end{array}$ & $\begin{array}{l}64,1 \\
18 \\
\end{array}$ & 23 \\
\hline 6 & Malaysia & $\begin{array}{l}2,3 \\
15 \\
\end{array}$ & $\begin{array}{l}9,4 \\
18 \\
\end{array}$ & $\begin{array}{l}5,7 \\
/ 6 \\
\end{array}$ & 19 & $\begin{array}{l}82,3 \\
17 \\
\end{array}$ & $\begin{array}{l}68,0 \\
18 \\
\end{array}$ & $\begin{array}{l}65,3 \\
17 \\
\end{array}$ & 22 \\
\hline 7 & Thailand & $\begin{array}{l}2,8 \\
19 \\
\end{array}$ & \begin{tabular}{|l}
7,9 \\
14 \\
\end{tabular} & $\begin{array}{l}6,5 \\
19 \\
\end{array}$ & 22 & $\begin{array}{l}64,2 \\
/ 10 \\
\end{array}$ & $\begin{array}{l}59,2 \\
/ 14\end{array}$ & $\begin{array}{l}53,2 \\
/ 12\end{array}$ & 36 \\
\hline 8 & Rep. Korea & $\begin{array}{l}2,9 \\
/ 11\end{array}$ & $\begin{array}{l}8 \\
15 \\
\end{array}$ & $\begin{array}{l}5,9 \\
17 \\
\end{array}$ & 23 & $\begin{array}{l}82,4 \\
16 \\
\end{array}$ & $\begin{array}{l}76,0 \\
17 \\
\end{array}$ & $\begin{array}{l}79,8 \\
16 \\
\end{array}$ & 19 \\
\hline 9 & Australia & $\begin{array}{l}2,8 \\
/ 10 \\
\end{array}$ & $\begin{array}{l}10,4 \\
/ 10 \\
\end{array}$ & $\begin{array}{l}7,9 \\
/ 11 \\
\end{array}$ & 31 & $\begin{array}{l}94,7 \\
12 \\
\end{array}$ & $\begin{array}{l}95,9 \\
13 \\
\end{array}$ & $\begin{array}{l}95,0 \\
12 \\
\end{array}$ & 7 \\
\hline 10 & Mexico & $\begin{array}{l}4,3 \\
/ 12 \\
\end{array}$ & $\begin{array}{l}9,6 \\
19 \\
\end{array}$ & $\begin{array}{l}6,9 \\
/ 10 \\
\end{array}$ & 31 & $\begin{array}{l}61,5 \\
/ 12 \\
\end{array}$ & $\begin{array}{l}63,0 \\
/ 11 \\
\end{array}$ & $\begin{array}{l}36,9 \\
/ 19 \\
\end{array}$ & 42 \\
\hline 11 & China Mainland & $\begin{array}{l}2,6 \\
17 \\
\end{array}$ & $\begin{array}{l}17,8 \\
/ 18 \\
\end{array}$ & $\begin{array}{l}5,9 \\
/ 8 \\
\end{array}$ & 33 & $\begin{array}{l}57,4 \\
/ 13 \\
\end{array}$ & $\begin{array}{l}45,4 \\
/ 17 \\
\end{array}$ & $\begin{array}{l}41,1 \\
/ 16 \\
\end{array}$ & 46 \\
\hline 12 & Philippines & $\begin{array}{l}4,3 \\
/ 13 \\
\end{array}$ & $\begin{array}{l}11,3 \\
/ 11 \\
\end{array}$ & $\begin{array}{l}8,4 \\
/ 12 \\
\end{array}$ & 36 & $\begin{array}{l}55,6 \\
/ 14 \\
\end{array}$ & $\begin{array}{l}49,9 \\
/ 16 \\
\end{array}$ & $\begin{array}{l}37,8 \\
/ 18 \\
\end{array}$ & 48 \\
\hline 13 & South Africa & $\begin{array}{l}5,9 \\
/ 14 \\
\end{array}$ & $\begin{array}{l}12,9 \\
/ 13\end{array}$ & $\begin{array}{l}11,7 \\
/ 15\end{array}$ & 42 & $\begin{array}{l}69,3 \\
18 \\
\end{array}$ & $\begin{array}{l}67,3 \\
19 \\
\end{array}$ & $\begin{array}{l}56,5 \\
19\end{array}$ & 26 \\
\hline 14 & Peru & $\begin{array}{l}2,6 \\
/ 8\end{array}$ & $\begin{array}{l}12,3 \\
/ 12\end{array}$ & $\begin{array}{l}21,5 \\
/ 24 \\
\end{array}$ & 44 & $\begin{array}{l}41,9 \\
/ 21\end{array}$ & $\begin{array}{l}62,3 \\
/ 12 \\
\end{array}$ & $\begin{array}{l}31,0 \\
/ 20 \\
\end{array}$ & 53 \\
\hline 15 & India & $\begin{array}{l}7,4 \\
/ 16 \\
\end{array}$ & $\begin{array}{l}17 \\
/ 17 \\
\end{array}$ & $\begin{array}{l}11,2 \\
/ 13 \\
\end{array}$ & 46 & $\begin{array}{l}54,0 \\
/ 15 \\
\end{array}$ & $\begin{array}{l}40,8 \\
/ 19 \\
\end{array}$ & $\begin{array}{l}54,7 \\
/ 11 \\
\end{array}$ & 45 \\
\hline 16 & Indonesia & $\begin{array}{l}7,5 \\
/ 17 \\
\end{array}$ & $\begin{array}{l}13,3 \\
/ 14 \\
\end{array}$ & $\begin{array}{l}14,30 \\
118 \\
\end{array}$ & 49 & $\begin{array}{l}44,2 \\
/ 20 \\
\end{array}$ & $\begin{array}{l}36,7 \\
/ 21 \\
\end{array}$ & $\begin{array}{l}29,2 \\
/ 22 \\
\end{array}$ & 63 \\
\hline 17 & Egypt & $\begin{array}{l}9 \\
16 \\
\end{array}$ & $\begin{array}{l}13,6 \\
/ 15\end{array}$ & $\begin{array}{l}12,50 \\
117\end{array}$ & 51 & $\begin{array}{l}38,9 \\
/ 23 \\
\end{array}$ & $\begin{array}{l}38,7 \\
/ 20 \\
\end{array}$ & $\begin{array}{l}49,6 \\
/ 14 \\
\end{array}$ & 57 \\
\hline 18 & Brazil & $\begin{array}{l}6,5 \\
/ 15\end{array}$ & $\begin{array}{l}16,4 \\
/ 16\end{array}$ & $\begin{array}{l}47,90 \\
/ 25\end{array}$ & 56 & $\begin{array}{l}53,0 \\
/ 16\end{array}$ & $\begin{array}{l}56,0 \\
/ 15\end{array}$ & $\begin{array}{l}46,4 \\
/ 15\end{array}$ & 46 \\
\hline 19 & Romania & $\begin{array}{l}8,6 \\
/ 18\end{array}$ & $\begin{array}{l}19,6 \\
/ 19\end{array}$ & $\begin{array}{l}17,80 \\
/ 21\end{array}$ & 58 & $\begin{array}{l}47,0 \\
/ 18\end{array}$ & $\begin{array}{l}65,3 \\
/ 10\end{array}$ & $\begin{array}{l}52,1 \\
/ 13\end{array}$ & 41 \\
\hline 20 & Russia & $\begin{array}{l}10,4 \\
/ 22 \\
\end{array}$ & $\begin{array}{l}27,3 \\
/ 24 \\
\end{array}$ & $\begin{array}{l}11,40 \\
114\end{array}$ & 60 & $\begin{array}{l}41,8 \\
/ 22 \\
\end{array}$ & $\begin{array}{l}42,3 \\
/ 18 \\
\end{array}$ & \begin{tabular}{|l|}
23,6 \\
$/ 24$ \\
\end{tabular} & 64 \\
\hline 21 & Vietnam & $\begin{array}{l}9,3 \\
/ 21 \\
\end{array}$ & $\begin{array}{l}26,8 \\
/ 23 \\
\end{array}$ & $\begin{array}{l}11,80 \\
/ 16\end{array}$ & 60 & $\begin{array}{l}45,2 \\
/ 19\end{array}$ & $\begin{array}{l}28,9 \\
/ 23\end{array}$ & $\begin{array}{l}39,5 \\
/ 17 \\
\end{array}$ & 59 \\
\hline 22 & Argentina & $\begin{array}{l}10,7 \\
/ 23\end{array}$ & $\begin{array}{l}23,6 \\
/ 21\end{array}$ & $\begin{array}{l}16,20 \\
/ 20\end{array}$ & 64 & $\begin{array}{l}51,1 \\
/ 17\end{array}$ & $\begin{array}{l}23,6 \\
/ 24\end{array}$ & $\begin{array}{l}29,4 \\
/ 21\end{array}$ & 62 \\
\hline 23 & Turkey & $\begin{array}{l}13,1 \\
/ 25 \\
\end{array}$ & $\begin{array}{l}19,9 \\
/ 20\end{array}$ & $\begin{array}{l}14,40 \\
/ 19\end{array}$ & 64 & $\begin{array}{l}62,5 \\
/ 11 \\
\end{array}$ & $\begin{array}{l}60,5 \\
/ 13 \\
\end{array}$ & $\begin{array}{l}56,2 \\
/ 10\end{array}$ & 34 \\
\hline
\end{tabular}




\begin{tabular}{|c|c|c|c|c|c|c|c|c|c|}
\hline \multicolumn{10}{|c|}{$\begin{array}{l}\text { Selected Economies "Monetary Sphere's Stability (MSS)" } \\
\text { and "Quality of "Economic" Institutions (EIQ)" Ranks [5]. }\end{array}$} \\
\hline \multirow[t]{3}{*}{$\begin{array}{l}\text { Num. of } \\
\text { Place }\end{array}$} & \multirow[t]{3}{*}{ Economy } & \multicolumn{4}{|c|}{$\begin{array}{c}\text { MSS Average Annual Rank } \\
(2002-2013) \\
\end{array}$} & \multicolumn{4}{|c|}{$\begin{array}{c}\text { EIQ Average Annual Rank } \\
(2002-2013)\end{array}$} \\
\hline & & CPI & $B M$ & $L I R$ & $\begin{array}{c}\text { Overall / } \\
\text { Rank }\end{array}$ & $\overline{G E}$ & $R Q$ & $R L$ & $\begin{array}{c}\text { Overall / } \\
\text { Rank }\end{array}$ \\
\hline & & $\begin{array}{l}\text { Data/ } \\
\text { Place }\end{array}$ & $\begin{array}{l}\text { Data/ } \\
\text { Place }\end{array}$ & $\begin{array}{l}\text { Data/ } \\
\text { Place }\end{array}$ & $(3+4+5)$ & $\begin{array}{l}\text { Data/ } \\
\text { Place }\end{array}$ & $\begin{array}{l}\text { Data/ } \\
\text { Place }\end{array}$ & $\begin{array}{l}\text { Data/ } \\
\text { Place }\end{array}$ & $(7+8+9)$ \\
\hline 24 & Ukraine & $\begin{array}{l}9,1 \\
120\end{array}$ & $\begin{array}{ll}29,5 \\
/ 25\end{array}$ & $\begin{array}{l}17,80 \\
/ 22\end{array}$ & 67 & $\begin{array}{l}29,4 \\
124\end{array}$ & $\begin{array}{ll}32,5 \\
122\end{array}$ & $\begin{array}{l}25,3 \\
123\end{array}$ & 69 \\
\hline 25 & Nigeria & $\begin{array}{l}11,8 \\
124\end{array}$ & $\begin{array}{l}25,1 \\
/ 22\end{array}$ & $\begin{array}{l}18,10 \\
/ 23\end{array}$ & 69 & $\begin{array}{l}14,9 \\
/ 25\end{array}$ & $\begin{array}{l}20,6 \\
/ 25\end{array}$ & $\begin{array}{l}10,4 \\
/ 25\end{array}$ & 75 \\
\hline
\end{tabular}

TABLE II. SELECTED ECONOMIES FINAL RANKING

\begin{tabular}{|c|c|c|c|c|}
\hline \multicolumn{5}{|c|}{$\begin{array}{l}\text { Selected Economies ranking on Ranks: } \\
\text { “GDP average annual rates"; "Monetary Sphere's Stability (MSS)"; “Quality of Economic Institutions (EIQ)”. The Correlation } \\
\text { Coefficients of Economies' Ranks [5]. }\end{array}$} \\
\hline $\mathbf{N N}$ & Economy & $\begin{array}{l}\text { GDP average annual rates } \\
(\%) 2002-2013\end{array}$ & $\begin{array}{c}\text { MSS Rank } \\
\text { 2002-2013 }\end{array}$ & $\begin{array}{l}\text { EIQ Rank } \\
\text { 2002-2013 }\end{array}$ \\
\hline 1 & China Mainland & 10,2 & 33 & 46 \\
\hline 2 & Nigeria & 8,5 & 69 & 75 \\
\hline 3 & India & 7,3 & 46 & 45 \\
\hline 4 & Vietnam & 6,4 & 60 & 59 \\
\hline 5 & Peru & 6,2 & 44 & 53 \\
\hline 6 & Turkey & 6 & 64 & 34 \\
\hline 7 & Indonesia & 5,5 & 49 & 63 \\
\hline 8 & Philippines & 5,2 & 36 & 48 \\
\hline 9 & Malaysia & 5,1 & 19 & 22 \\
\hline 10 & Thailand & 4,5 & 22 & 36 \\
\hline 11 & Russia & 4,4 & 60 & 64 \\
\hline 12 & Egypt & 4,3 & 51 & 57 \\
\hline 13 & Argentina & 4,1 & 64 & 62 \\
\hline 14 & Korea Rep & 4,1 & 23 & 19 \\
\hline 15 & Brazil & 3,7 & 56 & 46 \\
\hline 16 & Romania & 3,7 & 58 & 41 \\
\hline 17 & Ukraine & 3,5 & 67 & 69 \\
\hline 18 & South Africa & 3,4 & 42 & 26 \\
\hline 19 & Australia & 3,1 & 31 & 7 \\
\hline 20 & Mexico & 2,4 & 31 & 42 \\
\hline 21 & US & 1,8 & 10 & 12 \\
\hline 22 & Britain & 1,5 & 12 & 8 \\
\hline 23 & Euro Area - Netherlands & 1 & 10 & 3 \\
\hline 24 & Japan & 0,8 & 3 & 15 \\
\hline 25 & Italy & $-0,2$ & 15 & 23 \\
\hline \multicolumn{3}{|c|}{$\begin{array}{l}\text { The Correlation Coefficient MSS and EIQ Ranks for All Selected Economies - } \\
\text { Total }\end{array}$} & \multicolumn{2}{|c|}{$\mathrm{R} 1=0,820167$} \\
\hline \multicolumn{3}{|c|}{$\begin{array}{l}\text { The Correlation Coefficient MSS and EIQ Ranks for All Selected Economies } \\
\text { except USA, Britain, Netherlands, Italy, Japan }\end{array}$} & \multicolumn{2}{|c|}{$\mathrm{R} 2=0,7$} \\
\hline \multirow{2}{*}{\multicolumn{3}{|c|}{$\begin{array}{l}\text { The Correlation Coefficient GDP average annual rates and MSS Ranks for All } \\
\text { Selected Economies - Total } \\
\text { The Correlation Coefficient GDP average annual rates and MSS Ranks for All } \\
\text { Selected Economies, except USA, Britain, Netherlands, Italy, Japan }\end{array}$}} & \multicolumn{2}{|c|}{$\mathrm{R} 3=0,547201$} \\
\hline & & & \multicolumn{2}{|c|}{$\mathrm{R} 4=0,107512$} \\
\hline \multicolumn{3}{|c|}{$\begin{array}{l}\text { The Correlation Coefficient GDP average annual rates and EIQ Ranks for All } \\
\text { Selected Economies - Total }\end{array}$} & \multicolumn{2}{|c|}{$\mathrm{R} 5=0,558698$} \\
\hline \multicolumn{3}{|c|}{$\begin{array}{l}\text { The Correlation Coefficient GDP average annual rates and EIQ Ranks for All } \\
\text { Selected Economies except USA, Britain, Netherlands, Italy, Japan }\end{array}$} & \multicolumn{2}{|c|}{$\mathrm{R} 6=0,313993$} \\
\hline
\end{tabular}

